\title{
Reconstruction of chronic thumb metacarpophalangeal radial collateral ligament injuries with free tendon grafts
}

\author{
Birkan Kibar, MD (D), Fahri Erdi Malkoç, MD (D) \\ Department of Orthopedics and Traumatology, Haydarpaşa Numune Training and Research Hospital, Istanbul, Turkey
}

Radial collateral ligament (RCL) injuries account for $10-40 \%$ of collateral ligament injuries of the thumb metacarpophalangeal (MP) joint. ${ }^{[1]}$ In acute tears, immobilization with plaster or splint is recommended. Complete tears are classified as grade 3 and denote a complete ligament tear with an absence of a solid end point on stress testing. In chronic grade 3 RCL injuries, a dorsoradial prominence occurs in the MP joint, resulting in weakness and pain during activity; the recent literature supports surgical treatment for this type of injury. ${ }^{[2]}$ There are many surgical treatment options for chronic tears, including RCL soft tissue advancement, reconstruction with the abductor pollicis brevis (APB), and free tendon grafts. ${ }^{[3-5]}$ In this study, we aimed to review our reconstruction method to treat chronic grade 3 thumb MP joint RCL tears using free tendon grafts. ${ }^{[6]}$

\section{PATIENTS AND METHODS}

This study was conducted at Haydarpaşa Numune Training and Research Hospital between May 2016 and July 2018. Adult patients who received a free tendon graft to treat chronic grade 3 thumb MP joint RCL injury were evaluated prospectively. Patients with incomplete bone maturation $(n=1)$, those who had additional injuries in the same extremity $(n=1)$, and

Received: August 01, 2019

Accepted: October 08, 2019

Published online: March 02, 2020

Correspondence: Birkan Kibar, MD. Haydarpaşa Numune Eğitim ve Araş̧ırma Hastanesi Ortopedi ve Travmatoloji Kliniği, El Cerrahisi Bölümü, 34668 Üsküdar, İstanbul, Türkiye

E-mail: birkankibar545454@gmail.com

Doi: 10.5606/ehc. 2020.70796

Citation: Kibar B, Malkoç FE. Reconstruction of chronic thumb metacarpophalangeal radial collateral ligament injuries with free tendon grafts. Jt Dis Relat Surg 2020;31(1):62-67.

\section{ABSTRACT}

Objectives: This study aims to review our reconstruction method to treat chronic grade 3 thumb metacarpophalangeal (MP) joint radial collateral ligament (RCL) tears using free tendon grafts.

Patients and methods: Thirteen male patients (mean age 41 years; range, 20 to 67 years) who received a free tendon graft to treat chronic grade 3 thumb MP joint RCL injury between May 2016 and July 2018 were evaluated prospectively. Patients with incomplete bone maturation $(n=1)$, those who had additional injuries in the same extremity $(n=1)$, and those who had previously undergone surgery on the same extremity $(n=1)$ were excluded. The Disabilities of the Arm, Shoulder and Hand (DASH) questionnaire was used for functional evaluation and the visual analog scale (VAS) was also used for pain evaluation.

Results: The decrease in DASH $(p=0.008)$ and VAS $(p=0.03)$ scores and the increase in grip $(\mathrm{p}=0.031)$ and pinch strength $(p=0.006)$ between the preoperative and final follow-up values were statistically significant. No ulnar deviation was observed in $\mathrm{X}$-rays at the final examination, nor was MP joint radial instability observed under ulnar stress during clinical examinations. In two patients, 1 and $1.5 \mathrm{~mm}$ palmar subluxation was observed at final examination. The other 11 patients had no palmar subluxation at final examination.

Conclusion: Although ours is a more complicated method compared to other reconstruction approaches, we think that it is a suitable alternative for achieving joint stability and good functional results.

Keywords: Chronic, graft, metacarpophalangeal joint, radial collateral ligament, tendon, thumb, traumatic.

those who had previously undergone surgery on the same extremity $(\mathrm{n}=1)$ were excluded. The study protocol was approved by the Haydarpaşa Numune Training and Research Hospital Ethics Committee (No: 2016/79). A written informed consent was obtained from each patient. The study was conducted in accordance with the principles of the Declaration of Helsinki.

During the study period, all chronic grade 3 thumb RCL injuries were operated with the same technique. One patient was lost during the follow-up period, and a total of 13 male patients (mean age 41 years; 
range, 20 to 67 years) were included in the study. All patients presented with pain in the MP joint when grasping objects and a dorsoradial prominence. Nine of the patients underwent splint treatment for three-six weeks, while the other four patients had no treatment. When ulnar stress was applied to the MP joint at a flexion of $30^{\circ}$, the presence of instability of more than $30^{\circ}$ or $15^{\circ}$ from the contralateral side and not arriving at an endpoint were classified as radial instability. In preoperative lateral X-rays of MP joint, volar displacement of the proximal phalanx by more than $3 \mathrm{~mm}$ relative to the metacarpal was classified as palmar subluxation. ${ }^{[7]}$ All patients in the study had palmar subluxation (mean percentage of palmar subluxation: $32.3 \%$; standard deviation [SD]: 3.2; range, 28 to 38 ) and radial instability. There was no evidence of MP joint osteoarthritis in the preoperative radiographs. All patients were operated on by the senior author under general or axillary block anesthesia. Our surgical technique is a mirror image of that of Glickel. ${ }^{[8]}$

One hour before the surgery, $1 \mathrm{~g}$ of cefazolin was administered to all patients. All patients were placed on the operating table in a supine position. A pneumatic tourniquet was used at a pressure of $250 \mathrm{mmHg}$. A lazy S incision was performed along the radial side of the MP joint. The skin flaps were elevated and the superficial branch of the radial nerve was protected. A longitudinal incision was performed to the abductor aponeurosis from the volar to the extensor pollicis brevis (EPB) leaving a 1-2 mm dorsal rim for closure. The abductor aponeurosis was elevated to volar, and the RCL was examined. The RCL was fibrotic, weak and shortened in all cases and thus not suitable for primary repair. After the RCL remnants were removed, two holes were drilled in the radial side of the proximal phalanx with a $2.7 \mathrm{~mm}$ drill at the 7 and 11 o'clock positions. A hole to the metacarpal neck was then opened to $1-2 \mathrm{~mm}$ dorsal of the central axis with a single cortex pattern. After joint reduction, the MP joint was fixed with 1.2 or $1.4 \mathrm{~mm}$ Kirschner (K) wire, obliquely from ulnar side of the proximal phalanx to radial side of the distal metacarpal, at MP joint $15-20^{\circ}$ flexion. A tendon graft was then harvested, palmaris longus (PL) was preferred and the slip of the flexor carpi radialis (FCR) was used as an alternative. The PL was used in 11 patients and the FCR in two. Sutures were placed to two free ends of the tendon graft, the two limbs were passed through the holes in the proximal phalanx and then through the hole in the metacarpal neck. A small counter-incision was performed in the ulnar side of the metacarpal, and the suture ends were passed through the ulnar cortex of metacarpal using straight needles. The graft was placed under tension, and the sutures were tied down over the ulnar cortex. The dorsal capsule and EPB tendon were found avulsed in all thumbs and the dorsal capsule and EPB tendon were repaired to dorsal edge of the tendon graft, the skin was closed, and a thumb spica cast was employed (Figure 1).

Thumb spica cast and the K-wire were removed after six weeks. After that, active and passive motion of the interphalangeal (IP), MP, and wrist joints was initiated and gradually progressed. The patients consulted the physical medicine and rehabilitation unit of our hospital and embarked on a hand rehabilitation program for one hour/day, five days/week, including neuromuscular electric stimulation and stretching exercises. Thenar muscle and pinch strengthening exercises were initiated after 12 weeks until painless, functional recovery was achieved. Full activity, including contact sports were allowed at least five months postoperatively.

Recent examinations were performed by a physical therapy and rehabilitation specialist in our hospital, experienced in hand therapy, independent of the surgical team. The range of motion (ROM) for the IP and MP joints was measured using a finger goniometer. The Disabilities of the Arm, Shoulder, and Hand (DASH) questionnaire was used for functional evaluation and the visual analog scale (VAS) was also used for pain evaluation..$^{[9,10]}$ A Saehan hydraulic hand dynamometer (Saehan Corporation, Changwon, South Korea) was used to measure the patient's grip strength. A pinch gauge was used for key pinch strength measurements.

\section{Statistical analysis}

Analyses were performed using the IBM SPSS version 22.0 statistical software (IBM Corp., Armonk, NY, USA). Paired sample t-tests were used to compare the parameters, considering $\mathrm{p}<0.05$ statistically significant.

\section{RESULTS}

Follow-up data were recorded at a minimum of one year with an average of $21.6 \pm 8.9$ months; range, 12 to 40). The mean interval between the injury and surgery was $10 \pm 1.5$ weeks; range, 8 to 13 ). The dominant side was affected in seven patients $(53 \%)$. The right side of eight and the left side of five patients were affected. The cause of injury was a fall for six patients, injury during a sporting activity for three patients, a workplace accident for two patients, a traffic accident for one patient, and assault for one patient. 

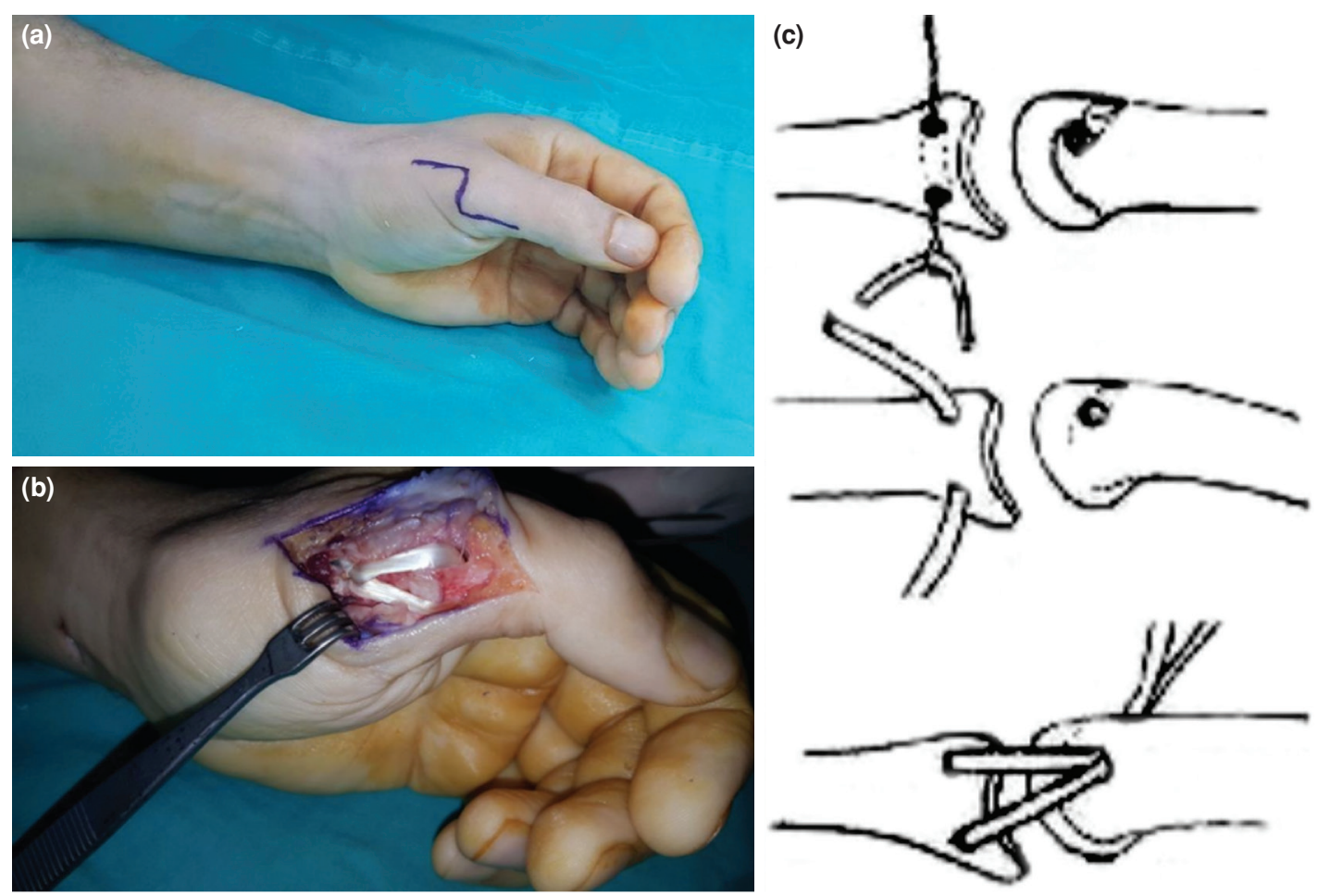

FIGURE 1. Intraoperative photograph of a 53-year-old male patient with chronic radial collateral ligament injury, (a) incision and (b) reconstruction with palmaris longus. (c) Two holes were drilled in radial side of proximal phalanx with a $2.7 \mathrm{~mm}$ drill at seven and 11 o'clock positions for passage of tendon graft, a hole to metacarpal neck was then opened to 1-2 mm dorsal of central axis which both limbs of free tendon graft are passed. Suture ends were passed through ulnar cortex of metacarpal using straight needles. Holes need to be separated sufficiently to preserve a solid bony bridge between them. Graft was placed under tension, and sutures were tied down over ulnar cortex.

At the final follow-up, MP joint extension (mean: $9.3 \pm 7.3^{\circ}$; range, 0 to $25^{\circ}$ ) did not change significantly compared to preoperative values (mean: $11 \pm 3.8^{\circ}$; range, 0 to $30^{\circ} ; \mathrm{p}=0.579$ ), while MP joint flexion (mean: $71 \pm 8.3^{\circ}$; range, 55 to $82^{\circ}$ ) was significantly lower compared to preoperative values (mean: $79 \pm 4.7^{\circ}$; range, 72 to $88^{\circ} ; \mathrm{p}=0.023$ ). There was no significant difference in IP joint extension between preoperative and final follow-up. At the final follow-up, IP joint flexion (mean: $73 \pm 10.2^{\circ}$; range, 60 to $90^{\circ}$ ) was

\begin{tabular}{|c|c|c|c|c|c|c|c|c|}
\hline \multicolumn{9}{|c|}{$\begin{array}{c}\text { TABLE I } \\
\text { Summary of functional results }\end{array}$} \\
\hline & \multicolumn{2}{|c|}{ Preoperative } & \multicolumn{2}{|c|}{ Postoperative } & \multirow[b]{2}{*}{$p^{*}$} & \multicolumn{2}{|c|}{ Healthy side } & \multirow[b]{2}{*}{$p^{\star *}$} \\
\hline & Mean $\pm S D$ & Range & Mean $\pm S D$ & Range & & Mean \pm SD & Range & \\
\hline Mean DASH & $20.3 \pm 8.5$ & $8-33$ & $9 \pm 7.1$ & $0-22$ & 0.008 & & & \\
\hline Mean visual analog scale & $4.4 \pm 1.7$ & $2-7$ & $1.3 \pm 1$ & $0-3$ & 0.003 & & & \\
\hline Mean grip strength (kg) & $31.1 \pm 8.5$ & $19-48$ & $36.3 \pm 10.6$ & $22-52$ & 0.031 & $40.1 \pm 9.3$ & $27-54$ & 0.219 \\
\hline Mean pinch strength (kg) & $3.3 \pm 1.2$ & $1.5-5.4$ & $6.1 \pm 1.7$ & $2.7-8.9$ & 0.006 & $6.8 \pm 1.4$ & $3.7-9.1$ & 0.424 \\
\hline Mean MP joint extension & $11 \pm 3.8^{\circ}$ & $0-30$ & $9.3 \pm 7.3^{\circ}$ & $0-25^{\circ}$ & 0.579 & $11.5 \pm 6.2^{\circ}$ & $0-30^{\circ}$ & 0.461 \\
\hline Mean MP joint flexion & $79 \pm 4.7^{\circ}$ & $72-88^{\circ}$ & $71 \pm 8.3^{\circ}$ & $55-82^{\circ}$ & 0.023 & $80 \pm 4^{\circ}$ & $75-90^{\circ}$ & 0.016 \\
\hline Mean IP joint extension & $5 \pm 4^{\circ}$ & $0-10^{\circ}$ & $4.8 \pm 4.7^{\circ}$ & $0-10^{\circ}$ & 0.895 & $5 \pm 4.3^{\circ}$ & $0-10^{\circ}$ & 0.912 \\
\hline Mean IP joint flexion & $81 \pm 6.4^{\circ}$ & $70-90^{\circ}$ & $73 \pm 10^{\circ}$ & $60-90^{\circ}$ & 0.039 & $81.9 \pm 5.9^{\circ}$ & $70-90^{\circ}$ & 0.024 \\
\hline
\end{tabular}



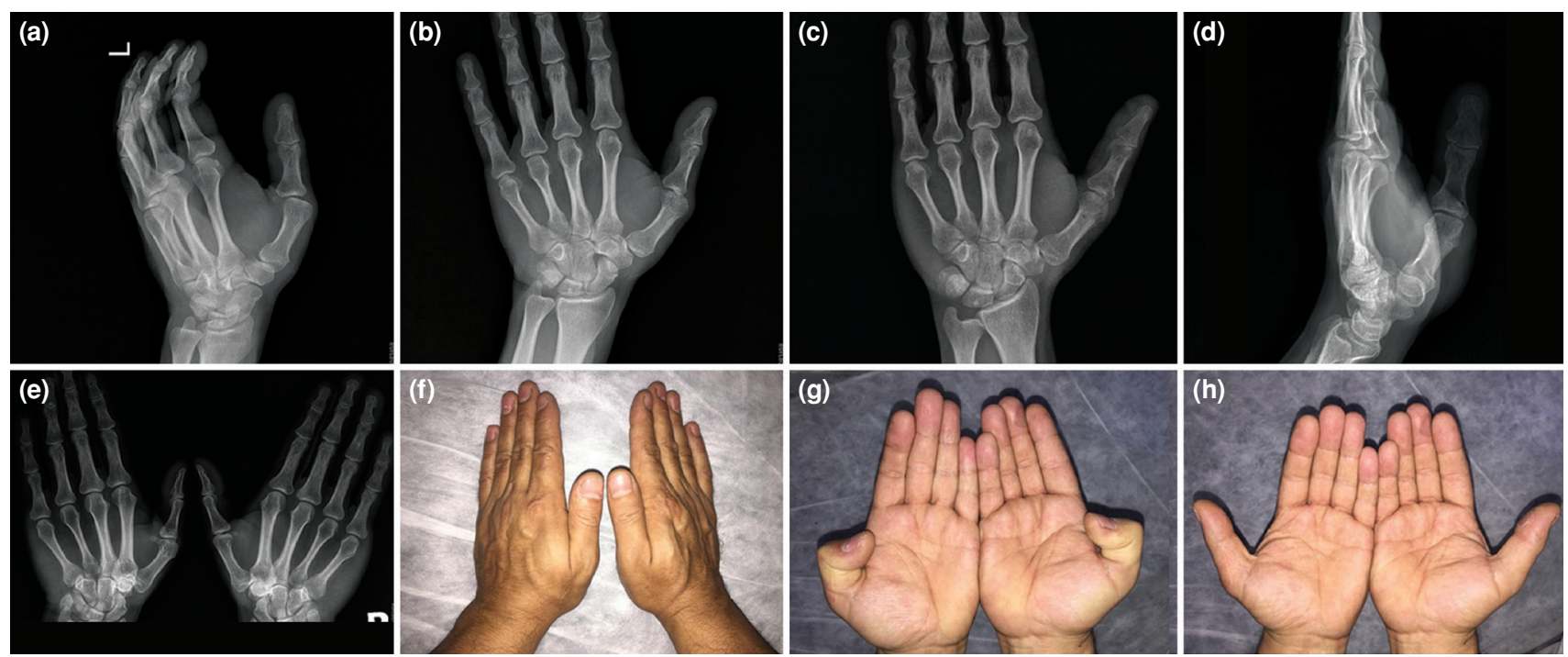

FIGURE 2. Preoperative radiographs of a 40-year-old male patient, (a, b) posterioanterior and lateral radiographs revealed palmar subluxation and ulnar deviation of proximal phalanx, (c) lateral radiograph of patient after Kirschner wire was removed, (d, e) postoperative first year radiographs of same patient, ulnar deviation appeared to have improved, palmar subluxation was partially improved, (f-h) postoperative first year clinical pictures of same patient.

significantly lower compared to preoperative values (mean: $81 \pm 6.4^{\circ}$; range, 70 to $90^{\circ} ; \mathrm{p}=0.039$ ). The decrease in DASH and VAS scores and the increase in grip and pinch strength between the preoperative and final follow-up values were statistically significant. Details are provided in Table I.

No ulnar deviation was observed in X-rays at the final examination, nor was MP joint radial instability observed under ulnar stress during clinical examinations. In two patients, palmar subluxations were not seen after K-wires were removed, but 1 and $1.5 \mathrm{~mm}$ palmar subluxation was observed at final examination. The other 11 patients had no palmar subluxation at final examination (Figure 2). No patient experienced infection, digital nerve injury, or hypertrophic scarring. No secondary surgery was required for any patient. All patients returned to their previous work or sporting activities and were satisfied with the treatment.

\section{DISCUSSION}

Injuries to the RCL are relatively uncommon. Typically, a classical ligament injury scheme is applied, while in grade 3 injuries, the ligament is completely torn, meaning there is no endpoint. Although there is no standard for differentiating between acute and chronic injury, a palmar subluxation of the proximal phalanx to the metacarpal of more than $3 \mathrm{~mm}$, as observed on lateral X-rays, is a sign for chronic injury, with palmar subluxation present in $68-85 \%$ of all chronic cases. ${ }^{[11]}$ In chronic cases, it is difficult to repair the RCL directly, so reconstruction is performed. Smith ${ }^{[12]}$ recommended reconstruction for cases over three weeks.

Many different reconstruction techniques have been proposed. In general, two reconstruction methods are applied: free tendon grafts and reconstruction using local muscles and tendons. The APB is not disrupted when a free tendon graft is employed, and the position of the graft is flexible. Local tendons have better blood supply; therefore, no additional incisions are required. However, to our knowledge, there have been no studies comparing these two methods. We preferred to employ free tendon grafts in our study. Coyle treated 45 patients with grade 3 RCL injuries with soft tissue sleeve advancement with bony reattachment without additional soft tissue reinforcement, reporting that 33 of the patients were symptom-free, 35 had a normal pinch and grip strength, and 30 achieved a normal ROM, thus this method was recommended for acute, chronic, and late grade 3 injuries. ${ }^{[1]}$ In addition, McDermott et al. ${ }^{[13]}$ applied a bone anchor to attach an avulsed ligament in five patients with chronic RCL injuries. All patients returned to daily living activities, and no patient had pain. Grip, key pinch, and MP joint ROM values were close to those exhibited by the uninjured side. ${ }^{[13]}$ Horch et al. ${ }^{[2]}$ applied the advancement of the tendinous part of the APB in nine patients with chronic RCL injuries, recommending the technique 
due to its simple nature, favorable results, and high patient satisfaction. Similarly, Camp et al. ${ }^{[4]}$ treated eight patients with chronic RCL injuries using APB distal advancement, with three patients experiencing mild pain and four patients having limited MP joint motion, and they reported that the method provides functional and clinical stability. Finally, Iba et al. ${ }^{[7]}$ applied the advancement and transfer of a half-slip of the APB tendon in eight patients with chronic RCL injuries, with a stable MP joint obtained in all patients, no patient experiencing ongoing symptoms, and a mean MP joint flexion loss of $6^{\circ}$.

In our study, patients with chronic thumb RCL injuries were treated with a free tendon graft, and a stable MP joint was obtained in all patients, with pinch and grip strength increasing significantly. In previous studies, acute and chronic tears, or different surgical techniques were evaluated within the same study, or evaluated with ulnar collateral ligament tears. Breek et al. ${ }^{[14]}$ applied free tendon grafts to 70 patients with acute and chronic MP joint injuries; 18 of these patients had a RCL injury, and, although $1 / 3$ of these patients had limited MP joint motion, it did not affect the functional results. They thus recommended the technique for all acute and chronic cases that did not respond to conservative treatment. In addition, Catalano et al. ${ }^{[15]}$ treated $26 \mathrm{RCL}$ injuries, 10 of which were chronic and reconstructed them with free tendon grafts. The mean MP joint motion was $57 \%$ of the uninjured side, and a stable MP joint was obtained in all patients, with eight excellent and two good results. Our results are consistent with these other studies and we believe that our proposed method improved the functional results.

Previous studies have reported the loss of MP joint flexion after RCL reconstruction; however, this loss did not lead to a functional deficiency. ${ }^{[1,5-7]}$ In our study, there was a decrease in flexion in the MP and IP joints, while this did not cause clinical dysfunction, and all patients returned to work and/or sporting activities. The loss of flexion may be caused by overtensioning the reconstructed tendon, although the graft must be tight in order to obtain stability. In addition, graft contraction and continuation of palmar subluxation may also cause joint stiffness. Cates et al. ${ }^{[16]}$ reconstructed chronic RCL rupture with free PL autograft and biotenodesis screw, injected botulinum toxin to adductor pollicis muscle before starting physical therapy to protect RCL reconstruction from deforming force of adductor pollicis and start earlier motion, and reported that botulinum toxin may decrease the period of immobilization.
The small size of the patient group, the absence of a control group and performing examinations in the same postoperative period were weaknesses of the research design.

In conclusion, although ours is a more complicated method compared to other reconstruction approaches, we think that it is a suitable alternative for achieving joint stability and good functional results.

\section{Declaration of conflicting interests}

The authors declared no conflicts of interest with respect to the authorship and/or publication of this article.

\section{Funding}

The authors received no financial support for the research and/or authorship of this article.

\section{REFERENCES}

1. Coyle MP Jr. Grade III radial collateral ligament injuries of the thumb metacarpophalangeal joint: treatment by soft tissue advancement and bony reattachment. J Hand Surg Am 2003;28:14-20.

2. Horch RE, Dragu A, Polykandriotis E, Kneser U. Radial collateralligament repair of the thumb metacarpophalangeal joint using the abductor pollicis brevis tendon. Plast Reconstr Surg 2006;117:491-6.

3. Melone CP Jr, Beldner S, Basuk RS. Thumb collateral ligament injuries. An anatomic basis for treatment. Hand Clin 2000;16:345-57.

4. Camp RA, Weatherwax RJ, Miller EB. Chronic posttraumatic radial instability of the thumb metacarpophalangeal joint. J Hand Surg Am 1980;5:221-5.

5. Durham JW, KhuriS, Kim MH. Acute and late radial collateral ligament injuries of the thumb metacarpophalangeal joint. J Hand Surg Am 1993;18:232-7.

6. Atik OŞ. Every new technique either conservative or surgical is good? Eklem Hastalik Cerrahisi 2019;30:183-4.

7. Iba K, Wada T, Hiraiwa T, Kanaya K, Oki G, Yamashita T. Reconstruction of chronic thumb metacarpophalangeal joint radial collateral ligament injuries with a half-slip of the abductor pollicis brevis tendon. J Hand Surg Am 2013;38:1945-50.

8. Glickel SZ. Thumb metacarpophalangeal joint ulnar collateral ligament reconstruction using a tendon graft. Tech Hand Up Extrem Surg 2002;6:133-9.

9. Cooney WP, Bussey R, Dobyns JH, Linscheid RL. Difficult wrist fractures. Perilunate fracture-dislocations of the wrist. Clin Orthop Relat Res 1987;214:136-47.

10. Hudak PL, Amadio PC, Bombardier C. Development of an upper extremity outcome measure: the DASH (disabilities of the arm, shoulder and hand) [corrected]. The Upper Extremity Collaborative Group (UECG) Am J Ind Med 1996;29:602-8.

11. Edelstein DM, Kardashian G, Lee SK. Radial collateral ligament injuries of the thumb. J Hand Surg Am 2008;33:760-70.

12. Smith RJ. Post-traumatic instability of the metacarpophalangeal joint of the thumb. J Bone Joint Surg Am 1977;59:14-21. 
13. McDermott TP, Levin LS. Suture anchor repair of chronic radial ligament injuries of the metacarpophalangeal joint of the thumb. J Hand Surg Br 1998;23:271-4.

14. Breek JC, Tan AM, van Thiel TP, Daantje CR. Free tendon grafting to repair the metacarpophalangeal joint of the thumb. Surgical techniques and a review of 70 patients. J Bone Joint Surg [Br] 1989;71:383-7.
15. Catalano LW 3rd, Cardon L, Patenaude N, Barron OA, Glickel SZ. Results of surgical treatment of acute and chronic grade III [corrected] tears of the radial collateral ligament of the thumb metacarpophalangeal joint. J Hand Surg Am 2006;31:68-75.

16. Cates RA, Brault JS, Kakar S. Botox and Thumb MCP Radial Collateral Ligament Reconstruction. J Wrist Surg 2018;7:156-9. 